\title{
Highly Chemoselective NH- and O-Transfer to Thiols Using Hypervalent lodine Reagents: Synthesis of Sulfonimidates and Sulfonamides
}

Arianna Tota, ${ }^{\ddagger, \S}$ Sahra St John-Campbell, ${ }^{\dagger, \S}$ Edward L. Briggs, ${ }^{\dagger}$ Gala Ogalla Estévez, ${ }^{\dagger}$ Michelle Afonso, ${ }^{\dagger}$

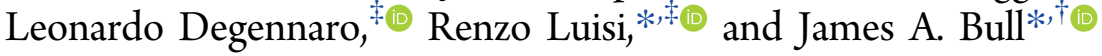

${ }^{\dagger}$ Department of Chemistry, Imperial College London, South Kensington, London SW7 2AZ, United Kingdom

${ }^{\ddagger}$ Department of Pharmacy-Drug Sciences, University of Bari, “A. Moro” Via E, Orabona 4, Bari 70125, Italy

\section{Supporting Information}

ABSTRACT: Aryl thiols can be selectively converted to sulfonimidates or sulfonamides with three new $S-X$ connections being made selectively in one pot. Using hypervalent iodine reagents in the presence of ammonium carbamate, $\mathrm{NH}$ - and $\mathrm{O}$ groups are transferred under mild and practical conditions. Reducing the loading of ammonium carbamate changed the product distribution, converting the sulfonimidate to the sulfonamide. Studies into the possible intermediate species are

presented, suggesting that multiple pathways may be possible via sulfinate esters, or related intermediates, with each species forming the same products.
$\mathrm{F}$ unctional groups that contain $\mathrm{S}-\mathrm{O}$ and $\mathrm{S}-\mathrm{N}$ linkages comprise some of the most useful motifs used in medicinal chemistry. ${ }^{1}$ Sulfonamides are the most common and are widely found in marketed drug compounds. Recently, sulfoximines have emerged as valuable groups for medicinal chemistry as chiral alternatives to sulfones ${ }^{2}$ and have progressed into clinical trials in several compounds. ${ }^{3}$ These have seen considerable synthetic advances in recent years, ${ }^{4}$ with developments in sulfoxide imination ${ }^{5}$ and sulfide imination, followed by oxidation. ${ }^{6}$ Sulfonimidamides, aza-analogues of sulfonamides, have also become recently appreciated for potential in medicinal chemistry and agrochemistry. ${ }^{7}$ Related sulfonimidates are less studied ${ }^{8}$ and are more reactive at $S$ through displacement of the alkoxide. This has led to application as sulfoximine precursors on reaction with organometallics, ${ }^{9}$ and in materials science as monomers for polymerization. ${ }^{10}$ They are also reactive by $S_{N} 2$ at the alkoxy carbon with the sulfur group as an effective leaving group affording a sulfonamide. ${ }^{11,12}$

Here, we report the selective formation of sulfonimidates through a highly chemoselective $\mathrm{NH}, \mathrm{O}$, and OR transfer to aryl thiols using hypervalent iodine reagents. A modified set of conditions affords sulfonamides, proceeding through the sulfonimidate as an intermediate. Preliminary studies into the order of atom transfer are presented, suggesting multiple pathways could afford the same products.

We recently reported facile conditions for the transfer of $\mathrm{NH}$ groups to sulfoxides to form $\mathrm{NH}$-sulfoximines, using bisacetoxyiodobenzene and ammonium carbamate (Figure 1). ${ }^{13}$ These conditions provided a mild and functional group tolerant approach to the unprotected sulfoximine group. An intermediate iminoiodinane species was invoked as the reactive

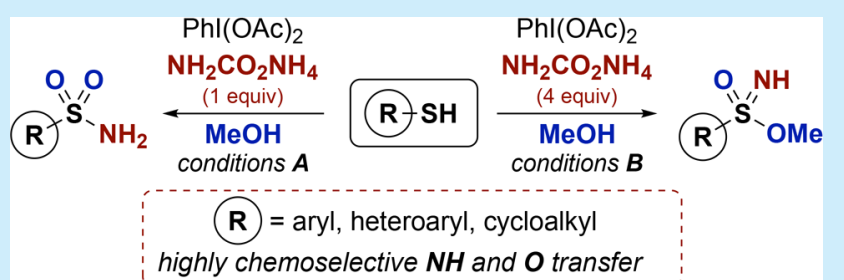
Luisi, Bull
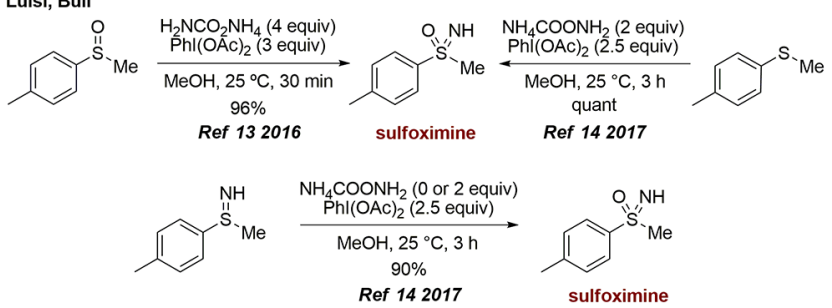

Stockman, Lücking

Stockman, L 16,2017
Ref

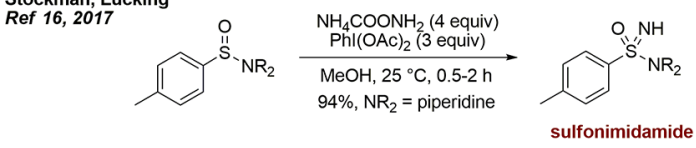

sulfoximine

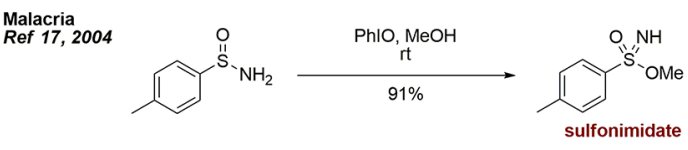

This work

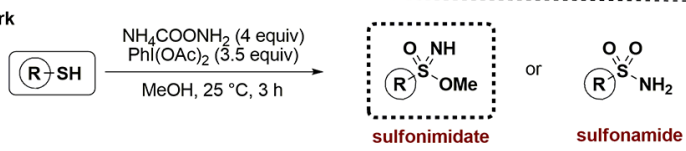

Figure 1. $\mathrm{NH}$ and $\mathrm{O}$ transfer to $\mathrm{S}$-groups to form sulfoximines, sulfondimidamides, and, in this work, sulfonimidates/sulfonamides.

$\mathrm{N}$-species. We then demonstrated that using sulfides, under essentially the same conditions, a chemoselective $\mathrm{NH}$ and $\mathrm{O}$ transfer occurred to generate sulfoximines directly. ${ }^{14,15}$ Applying the reaction conditions to diphenyl sulfilimine gave

Received: March 9, 2018

Published: April 13, 2018 
the conversion to the sulfoximine with or without the nitrogen source. Reboul reported related mechanistic and synthetic work at a similar time, which indicated that the $\mathrm{O}$ atom derived from methanol or acetate. ${ }^{13}$ Adopting these ammonium carbamate and bisacetoxyiodobenzene conditions, Stockman and Lücking reported $\mathrm{NH}$ transfer to sulfinamides to form sulfonimidamides with chemoselective $\mathrm{NH}$ transfer. ${ }^{16}$ Previously, the oxidation of sulfinamides to methyl sulfonimidates was demonstrated by Malacria using iodosobenzene in methanol to promote oxidation. $^{17}$

Given the value of these compound classes, we were intrigued by the potential for chemoselective transfer of $\mathrm{N}$ and/or $\mathrm{O}$ atoms to $\mathrm{S}$-functional groups starting at much lower oxidation levels. We intended to explore direct $\mathrm{S}-\mathrm{N}$ and $\mathrm{S}-\mathrm{O}$ bond formations from thiols targeting multiple bond formations in a single process to give valuable S-derivatives, and extend the N-transfer chemistry of hypervalent iodine reagents. ${ }^{18,19}$

Initially, we examined 4-tert-butylbenzenethiol 1a using a modification of our previously reported conditions (Scheme 1). We were delighted to find that a major product was formed corresponding to methyl sulfonimidate 2a. Primary sulfonamide $3 \mathbf{a}$ was also formed as a minor product.

Scheme 1. Initial Results Forming Sulfonimidate and Sulfonamide

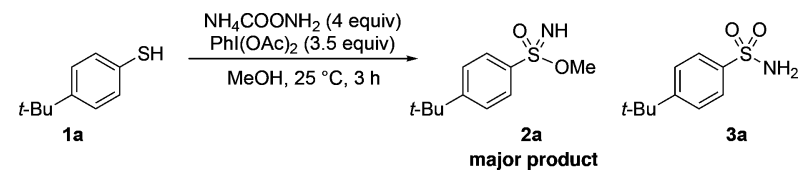

We subsequently optimized this process, varying the reaction conditions and equivalents of reagents. It was clear through these studies that running the reaction with fewer equivalents of ammonium carbamate gave increasing amounts of the sulfonamide. The best conditions to form sulfonimidate $\mathbf{2 a}$ used 4 equiv of ammonium carbamate and 4 equiv bisacetoxyiodobenzene. Unfortunately, despite considerable efforts to prevent formation of the sulfonamide product, it was always formed in small amounts and was inseparable by silica chromatography; hence, the sulfonimidate was not isolated cleanly. To improve separation and due to concern about the stability of the acid sensitive sulfonimidate on silica, we examined various stationary phases. ${ }^{20}$ Pleasingly, the use of neutral alumina was effective at removing the sulfonamide and afforded the pure sulfonimidate. Under these conditions the scope of the reaction was assessed, varying the arylthiol component (Scheme 2).

Using 4-tert-butylbenzene thiol, sulfonimidate 2 a was isolated in $65 \%$ yield. Various para-substituted examples were also successful $(\mathbf{2} \mathbf{b}-\mathbf{g})$, with generally higher yields with more electron-rich substrates. ortho-Substitution gave a somewhat reduced yield for the corresponding sulfonimidates $2 \mathbf{h}-\mathbf{k}$ (Scheme 2). Better yields were obtained for meta-substituted aromatic sulfonimidates $\mathbf{2} \mathbf{l}-\mathbf{n}$. More substituted aromatic thiols were found to be suitable for this transformation affording sulfonimidates 2o-q. Electron-rich heterosubstituted thiols such as thiophene-2-thiol furnished the corresponding sulfonimidate $\mathbf{2} \mathbf{r}$ in good yield.

Cyclohexanethiol was also suitable, furnishing sulfonimidate $2 \mathrm{~s}$ in $40 \%$ yield. Interestingly, the use of 2-mercaptobenzylalcohol gave the cyclic sulfonimidate $2 t$ through intramolecular
Scheme 2. Reaction Scope Forming Sulfonimidates with Aryl Thiols

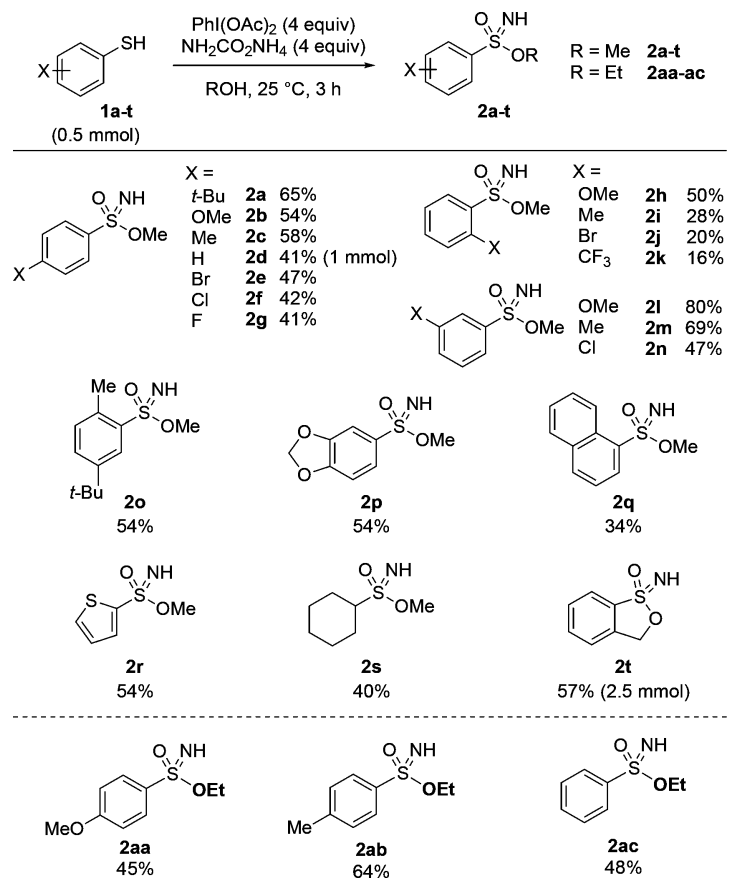

reaction of the benzylic alcohol in preference to the methanol solvent. ${ }^{21}$ Alternatively, running the reaction in EtOH afforded the corresponding ethyl sulfonimidates $2 \mathrm{aa}-\mathrm{ac}$. This protocol provides much more facile access to alkyl aryl-NH-sulfonimidates than has previously been available and avoids the preparation of sulfinamides.

At the same time, we optimized conditions for the direct formation of sulfonamides from the thiols (Scheme 3). ${ }^{22}$

Scheme 3. Reaction Scope Forming Sulfonamides with Aryl Thiols

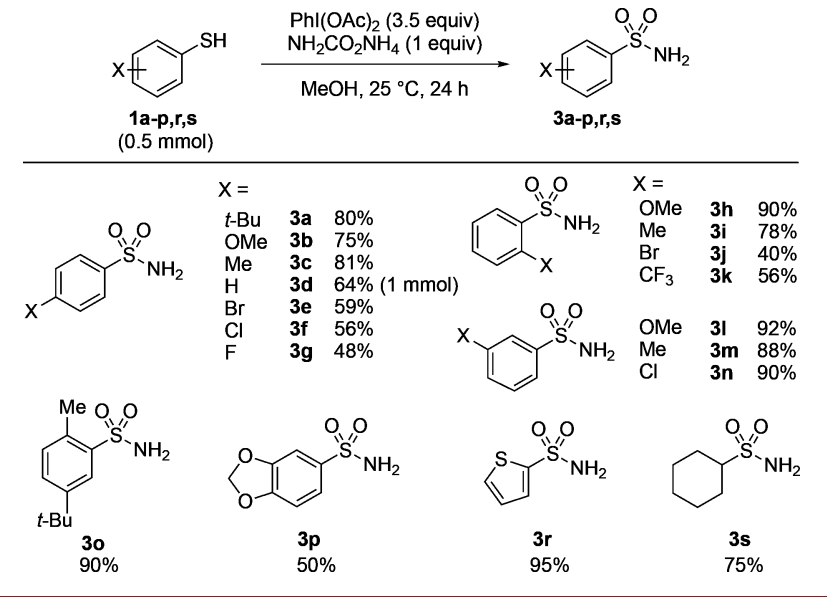

Running the reaction with only 1 equiv of ammonium carbamate and extending the reaction time to $24 \mathrm{~h}$, at $25{ }^{\circ} \mathrm{C}$, gave full conversion from the thiol to the sulfonamide, via the sulfonimidate. ${ }^{23}$ For this reaction, the yields were higher than those for the sulfonimidates and were again found to be dependent on the electronics of the aryl group. Sulfonamides $3 \mathbf{a}-\mathbf{n}$ were obtained in good to excellent yields from the corresponding aromatic thiols (Scheme 3). The protocol was successfully applied to thiols bearing polysubstituted aromatics 
(3o,p) as well as electron-rich heterocycle (3r) and alkyl moieties (3s).

Cyclic sulfonimidate $2 \mathrm{t}$ did not significantly convert to the sulfonamide under the longer reaction conditions, perhaps representing the stability of the cyclic system. Instead, and to provide insight into the mechanism, sulfonimidate $2 \mathrm{t}$ was treated with other nucleophiles (Scheme 4).

Scheme 4. Reaction of Sulfonimidates $2 t$ and $2 b$ with Nucleophiles

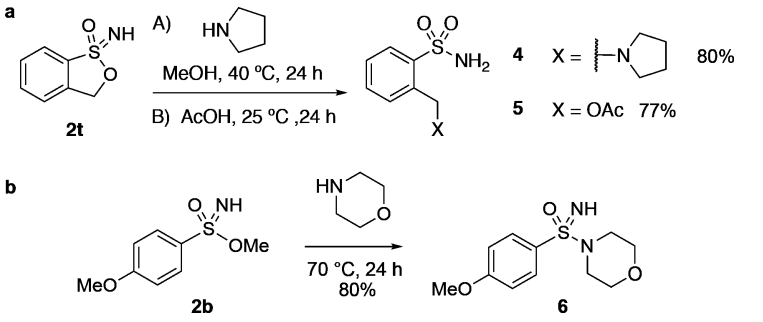

Treating the isolated sulfonimidate $2 \mathbf{t}$ with pyrrolidine in $\mathrm{MeOH}$ or with acetic acid resulted in ring opening to the sulfonamide products 4 and 5 , incorporating the nucleophile at the benzylic position. By contrast, treating the electron-rich 4methoxyphenyl sulfonimidate $\mathbf{2 b}$ with morpholine gave sulfonimidamide $\mathbf{6}$ in high yield with displacement at the sulfur center (Scheme $4 \mathrm{~b}$ ).

To provide insight into the reaction mechanism and order of events, we next examined other sulfur functional groups under related reaction conditions. Treating $\mathrm{PhSH}$ with oxidant alone quantitatively formed diphenyl disulfide $7 .^{24}$ The disulfide was converted to the methyl sulfinate ester 8 with an excess of oxidant (Scheme 5a). A mixture of 7 and $\mathbf{8}$ was obtained on

Scheme 5. Control Reactions

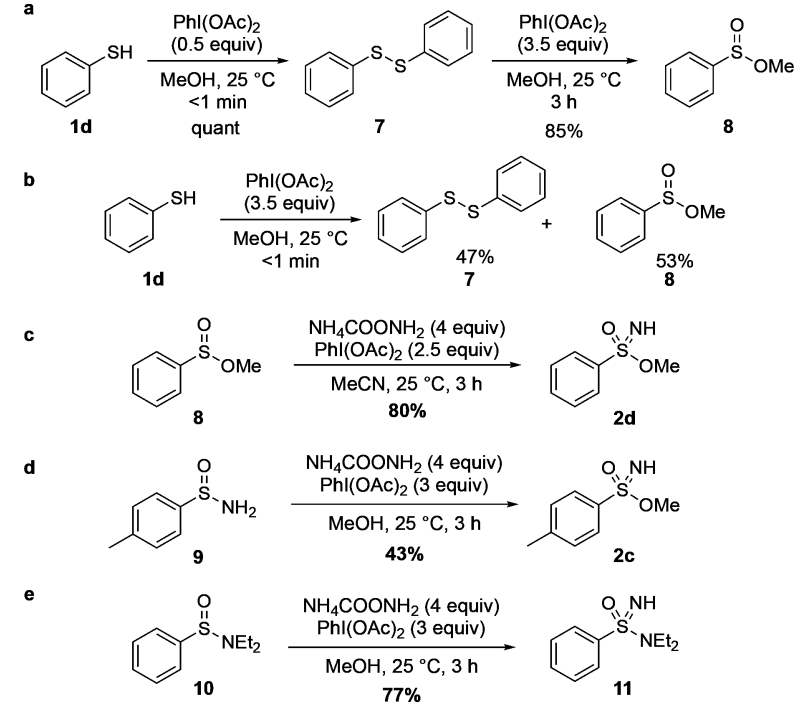

treating the thiol with 3.5 equiv of oxidant in $\mathrm{MeOH}$ (Scheme $5 b)$. Using methyl benzenesulfinate 8 as the substrate, sulfonimidate $\mathbf{2 d}$ was formed, using acetonitrile as the solvent (Scheme 5c). Importantly for the selectivity, the use of the reagents in the absence of the $\mathrm{N}$-source did not lead to oxidation of the sulfinate ester to the corresponding sulfonate ester. This provides a potentially valuable alternative route to sulfonimidates from sulfinate esters. In comparison to the
Malacria method, ${ }^{15}$ applying our conditions to a primary sulfinamide 9 also formed sulfonimidate 2c with small quantities of the sulfonamide (Scheme $5 \mathrm{~d}$ ). As recently reported by Lücking and Stockman, ${ }^{14}$ we also observed $\mathrm{NH}$ transfer in the presence of $\mathrm{N}, \mathrm{N}$-disubstituted sulfinamides (Scheme 5e). This is in contrast to the oxidation observed for the $\mathrm{NH}_{2}$ species.

In terms of the source of the $\mathrm{O}$-atom in the sulfonimidates, it is likely to be the $\mathrm{MeOH}$ solvent or acetate. This is consistent with Reboul's proposal for sulfide oxidation to form sulfoximines, ${ }^{15}$ a wich can also be compared with the conversion of sulfonimidate to sulfonamide by nucleophilic substitution.

To identify intermediate species, reactions using various sulfides were sampled and analyzed directly by GC and GCMS analysis. The reaction in all cases was fast, and intermediate species were not detected with the exception of the corresponding disulfide and sulfinate ester species. Only when investigating cyclohexanethiol as the substrate was another intermediate detected in the MS trace, which was putatively assigned as $\mathrm{cHexS}(\mathrm{NH}) \mathrm{OMe}$. Based on these results and our prior studies, the sequence reported in Scheme 6 is

Scheme 6. Plausible Reaction Sequence

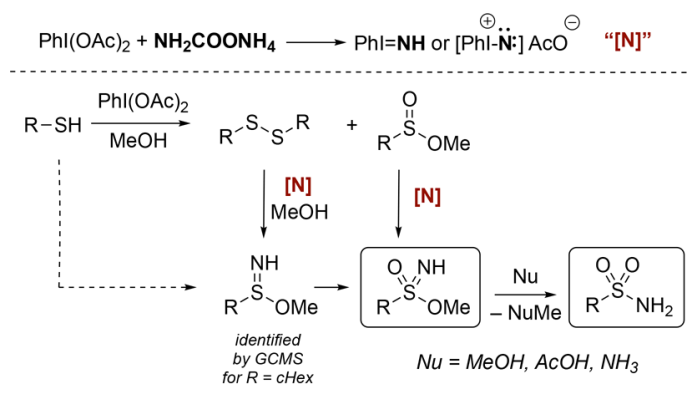

proposed. It is likely that multiple pathways may be followed, but that these are selectively converted to the same product. However, the exact sequence of events remains unclear.

In conclusion, sulfonimidates are selectively formed from thiols using ammonium carbamate and bisacetoxyiodobenzene in methanol. Conversion to sulfonamides occurs in the presence of lower ammonia concentrations and extended reaction times through substitution of the alkoxy group. Disulfides, sulfinates, and sulfinamides are all suitable precursors to sulfonimidates under these reaction conditions. Further studies are underway in our laboratories to elucidate mechanistic pathways and exploit these simple reagents for selective transformations.

\section{ASSOCIATED CONTENT}

\section{Supporting Information}

The Supporting Information is available free of charge on the ACS Publications website at DOI: 10.1021/acs.orglett.8b00788.

Experimental procedures, characterization data, and copies of ${ }^{1} \mathrm{H}$ and ${ }^{13} \mathrm{C}$ NMR spectra (PDF)

\section{AUTHOR INFORMATION}

\section{Corresponding Authors}

*E-mail: j.bull@imperial.ac.uk.

*E-mail: renzo.luisi@uniba.it. 


\section{ORCID $\odot$}

Leonardo Degennaro: 0000-0002-2187-9419

Renzo Luisi: 0000-0002-9882-7908

James A. Bull: 0000-0003-3993-5818

Author Contributions

${ }^{\S}$ A.T. and S.S.J.-C. contributed equally

Notes

The authors declare no competing financial interest.

\section{ACKNOWLEDGMENTS}

We gratefully acknowledge The Royal Society for a University Research Fellowship (to J.A.B.) and EPSRC [CAF to J.A.B. (EP/J001538/1), Impact Acceleration Account (EP/K503733/ 1), DTA Studentship (to S.S.J.-C. and E.L.B.)]. This research was supported by the project Laboratorio Sistema Code PONa300369 financed by MIUR, the University of Bari.

\section{REFERENCES}

(1) Ilardi, E. A.; Vitaku, E.; Njardarson, J. T. J. Med. Chem. 2014, 57, 2832.

(2) (a) Lücking, U. Angew. Chem., Int. Ed. 2013, 52, 9399. (b) Sirvent, J. A.; Lücking, U. ChemMedChem 2017, 12, 487. (c) Frings, M.; Bolm, C.; Blum, A.; Gnamm, C. Eur. J. Med. Chem. 2017, 126, 225.

(3) (a) Lücking, U.; Jautelat, R.; Krüger, M.; Brumby, T.; Lienau, P.; Schäfer, M.; Briem, H.; Schulze, J.; Hillisch, A.; Reichel, A.; Wengner, A. M.; Siemeister, G. ChemMedChem 2013, 8, 1067. (b) Foote, K. M.; Lau, A.; Nissink, J. W. M. Future Med. Chem. 2015, 7, 873.

(4) For recent reviews: (a) Bull, J. A.; Degennaro, L.; Luisi, R. Synlett 2017, 28, 2525. (b) Bizet, V.; Hendriks, C. M. M.; Bolm, C. Chem. Soc. Rev. 2015, 44, 3378.

(5) (a) Okamura, H.; Bolm, C. Org. Lett. 2004, 6, 1305. (b) Wang, J.; Frings, M.; Bolm, C. Chem. - Eur. J. 2014, 20, 966. (c) Mancheño, O. G.; Bolm, C. Org. Lett. 2006, 8, 2349. (d) Bizet, V.; Buglioni, L.; Bolm, C. Angew. Chem., Int. Ed. 2014, 53, 5639.

(6) For selected imination reactions of sulfides, see: (a) Lebel, H.; Piras, H. J. J. Org. Chem. 2015, 80, 3572. (b) Mancheño, O. G.; Bolm, C. Chem. - Eur. J. 2007, 13, 6674. (c) Cho, G. Y.; Bolm, C. Tetrahedron Lett. 2005, 46, 8007. (d) Armstrong, A.; Edmonds, I. D.; Swarbrick, M. E. Tetrahedron Lett. 2003, 44, 5335.

(7) Chinthakindi, P. K.; Naicker, T.; Thota, N.; Govender, T.; Kruger, H. G.; Arvidsson, P. I. Angew. Chem., Int. Ed. 2017, 56, 4100. (8) (a) Levchenko, E. S.; Derkach, N. Y.; Kirsanov, A. V. Zh. Obsh. Khim. 1960, 30, 1971. (b) Levchenko, E. S.; Markovskii, L. N.; Kirsanov, A. V. Zh. Org. Khim. 1967, 3, 1273; J. Org. Chem. USSR 1967, 3, 1439 .

(9) (a) Johnson, C. R.; Jonsson, E. U.; Wambsgans, A. J. Org. Chem. 1979, 44, 2061. (b) Reggelin, M.; Zur, C. Synthesis 2000, 2000, 1.

(10) Roy, A. K.; Burns, G. T.; Lie, G. C.; Grigoras, S. J. Am. Chem. Soc. 1993, 115, 2604.

(11) (a) Johnson, C. R.; Jonsson, E. U.; Bacon, C. C. J. Org. Chem. 1979, 44, 2055. (b) Maricich, T. J.; Jourdenais, R. A.; Albright, T. A. J. Am. Chem. Soc. 1973, 95, 5831. (c) Challis, B. C.; Iley, J. N. J. Chem. Soc., Perkin Trans. 2 1985, 2, 699.

(12) Maricich, T. J.; Allan, M. J.; Kislin, B. S.; Chen, A. I. T.; Meng, F. C.; Bradford, C.; Kuan, N. C.; Wood, J.; Aisagbonhi, O.; Poste, A.; Wride, D.; Kim, S.; Santos, T.; Fimbres, M.; Choi, D.; Elia, H.; Kaladjian, J.; Abou-Zahr, A.; Mejia, A. Synthesis 2013, 45, 3361.

(13) Zenzola, M.; Doran, R.; Degennaro, L.; Luisi, R.; Bull, J. A. Angew. Chem., Int. Ed. 2016, 55, 7203.

(14) Tota, A.; Zenzola, M.; Chawner, S. J.; St. John-Campbell, S.; Carlucci, C.; Romanazzi, G.; Degennaro, L.; Bull, J. A.; Luisi, R. Chem. Commun. 2017, 53, 348.

(15) Also see: (a) Lohier, J.-F.; Glachet, T.; Marzag, H.; Gaumont, A.-C.; Reboul, V. Chem. Commun. 2017, 53, 2064. (b) Xie, Y.; Zhou,
B.; Zhou, S.; Zhou, S.; Wei, W.; Liu, J.; Zhan, Y.; Cheng, D.; Chen, M.; Li, Y.; Wang, B.; Xue, X.; Li, Z. ChemistrySelect 2017, 2, 1620.

(16) Izzo, F.; Schäfer, M.; Stockman, R.; Lücking, U. Chem. - Eur. J. 2017, 23, 15189.

(17) (a) Leca, D.; Fensterbank, L.; Lacôte, E.; Malacria, M. Org. Lett. 2002, 4, 4093. (b) Felim, A.; Toussaint, A.; Phillips, C. R; Leca, D.; Vagstad, A.; Fensterbank, L.; Lacôte, E.; Malacria, M. Org. Lett. 2006, 8, 337. (c) Leca, D.; Song, K.; Amatore, M.; Fensterbank, L.; Lacôte, E.; Malacria, M. Chem. - Eur. J. 2004, 10, 906.

(18) (a) Yoshimura, A.; Zhdankin, V. V. Chem. Rev. 2016, 116, 3328. (b) Dauban, P.; Dodd, R. H. Synlett 2003, 1571.

(19) For selected developments in hypervalent iodine reagents with N-ligands, see: (a) Sandtorv, A. H.; Stuart, D. R. Angew. Chem., Int. Ed. 2016, 55, 15812. (b) Krasnova, L. B.; Hili, R. M.; Chernoloz, O. V.; Yudin, A. K. ARKIVOC 2005, iv, 26. (c) Souto, J. A.; Martínez, C.; Velilla, I.; Muñiz, K. Angew. Chem., Int. Ed. 2013, 52, 1324. (d) Lucchetti, N.; Scalone, M.; Fantasia, S.; Muñiz, K. Angew. Chem., Int. Ed. 2016, 55, 13335. (e) Marzag, H.; Schuler, M.; Tatibouët, A.; Reboul, V. Eur. J. Org. Chem. 2017, 2017, 896. (f) Wang, H.; Cheng, Y.; Becker, P.; Raabe, G.; Bolm, C. Angew. Chem., Int. Ed. 2016, 55, 12655.

(20) (a) Boultwood, T.; Affron, D. P.; Trowbridge, A. D.; Bull, J. A. J. Org. Chem. 2013, 78, 6632-6647. (b) Boultwood, T.; Affron, D. P.; Bull, J. A. J. Visualized Exp. 2014, 87, e51633. Using this protocol to check stability, we observed that the sulfonamide was significantly retained on the neutral alumina facilitating purification.

(21) Using technical grade 2-mercaptobenzylalcohol.

(22) For alternative synthesis of sulfonamides from thiols using $\mathrm{I}_{2}$ and TBHP, see: Feng, J.-B.; Wu, X.-F. Org. Biomol. Chem. 2016, 14, 6951.

(23) Resubjecting sulfonimidate $\mathbf{2 a}$ to the reaction conditions gave an $81 \%$ conversion to the sulfonamide. Presumably the increased ammonia concentration when using 4 equiv of ammonium carbamate buffers the acidic reaction conditions to reduce the $S_{N} 2$ alkylation of solvent or other nucleophiles as the first step of the rearrangement.

(24) Rapid oxidation of aryl thiols to disulfides has been reported using bisacetoxyiodobenzene in isopropanol: Rattanangkool, E.; Krailat, W.; Vilaivan, T.; Phuwapraisirisan, P.; Sukwattanasinitt, M.; Wacharasindhu, S. Eur. J. Org. Chem. 2014, 2014, 4795. 